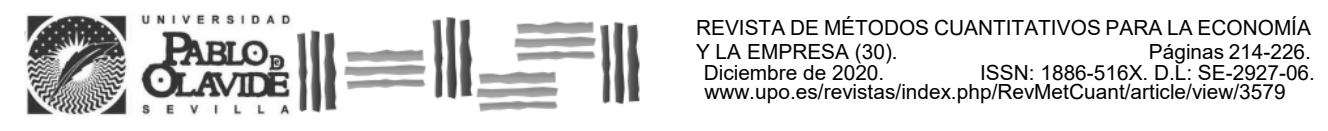

\title{
Un modelo cuantitativo de gobierno universitario
}

\author{
FRANK, LUIS \\ Universidad de Buenos Aires (Argentina) \\ Correo electrónico: frank@agro.uba.ar
}

\section{RESUMEN}

El trabajo explica, a través de un modelo cuantitativo, cómo se fijan las dedicaciones a la docencia y la investigación en universidades gobernadas por grupos de interés, tales como las universidades públicas argentinas. Para ello, se proponen funciones de utilidad docente y de costo político asumido por el Consejo Académico que gobierna la Universidad. La optimización simultánea de estas funciones conduce a un equilibrio general que satisface los intereses de todas las partes y garantiza la gobernabilidad de la institución, así como una asignación de tiempo óptima para las actividades de enseñanza e investigación.

Palabras clave: gobierno universitario, modelo, universidades públicas, Argentina.

Clasificación JEL: I21; I23.

MSC2010: 49-02. 


\title{
A quantitative model of university government
}

\begin{abstract}
The paper explains, through a quantitative model, how dedications to teaching and research are fixed in universities governed by interest groups, such as Argentines' public universities. To that end, utility functions of professors and a political cost function assumed by the Academic Council that governs the University are proposed. The simultaneous optimization of these functions leads to a general equilibrium that satisfies the interests of all parties and guarantees the governability of the institution, as well as an optimal time allocation to teaching and research activities.
\end{abstract}

Keywords: university government, model, public universities, Argentina.

JEL classification: I21; I23.

MSC2010: 49-02.

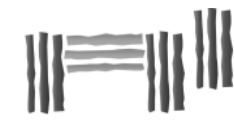

$=$<smiles>C#CC#CC#C</smiles><smiles>C#CC#C</smiles><smiles>C#CC#CC#C</smiles> 


\section{Introducción.}

En Argentina, el gobierno de las universidades nacionales se rige por la ley 24.521 de Educación Superior, los Estatutos Universitarios y los reglamentos dictados por los Consejos de cada institución. La Universidad de Buenos Aires (UBA), en particular, se rige por el Estatuto Universitario de 1960 (repuesto por decreto 157/83 y ley 23.068), por los reglamentos del Consejo Superior (CS) de la Universidad, entre los que se destaca el reglamento de provisión de cargos de profesores regulares (resolución CS 4362/12), y por los de los Consejos Directivos (CD) de sus 13 Facultades. La composición del Consejo Superiory de los Consejos Directivos (máximos órganos de gobierno) se basa en el principiode cogobierno entre profesores, graduados y alumnos, impulsado por la Reforma Universitaria de 1918. El CS de la UBA está integrado por 5 profesores, 5 graduados y 5 estudiantes, más los decanos de las 13 facultades y es presidido por el Rector. Los CD son órganos descentralizados y están constituidos por 8 profesores regulares, 4 graduados y 4 estudiantes, y son presididos por el Decano de la Facultad. Existen, además, órganos menores de gobierno, como las Juntas Departamentales, que replican esta estructura de cogobierno. La representación de estos tres claustros se halla sujeta a renovación periódica, cada dos años en el caso del claustro estudiantil y de graduados, y cada cuatro en el caso del claustro de profesores. Con alguna variante, todas las universidades nacionales siguen un esquema similar (para un panorama de la educación superior en Argentina, véase Fernández, 2002).

Las universidades nacionales son instituciones autónomas y autárquicas del Poder Ejecutivo Nacional (Convención Constituyente, 1994, art. 75 inc. 19), aunque el alcance de la autonomía es, a veces, motivo de debate entre juristas (Molina, 2014; Novelli, 2011). El concepto de autarquía en Derecho y Ciencias Políticas (ver por ejemplo García, 1995) se refiere a la facultad de un ente de administrarse a sí mismo, pero de acuerdo con una norma que le es impuesta, al contrario de la autonomía que se refiere la facultad del ente de darse sus propias instituciones y gobernarse por ellas. No obstante, en un fallo que fija jurisprudencia, la Corte Suprema de Justicia de la Nación CSJN (1996) definió que: "la autonomía universitaria consiste en que cada universidad nacional establezca su propio estatuto [...], elija sus autoridades, designe a los profesores, fije el sistema de nombramientos y disciplina interna". Es decir que, de acuerdo a la CSJN, el autogobierno y la capacidad de fijar su propio sistema de nombramiento de profesores son derechos constitucionales de las universidades nacionales.

La escasa bibliografía sobre la gobernanza de las universidades nacionales aborda la cuestión desde una perspectiva histórico-política (Buchbinder, 2010; Halperin, 2002; Unzué, 2012); normativa-institucional (Romera \& Puricelli, 1994, pp. 15-27; Marquís, 2010; Nosiglia \& Mulle, 2009, 2015); de fijación de estándares de calidad (CONEAU, 2007; Consejo de Universidades, 2015); incluso, desde una perspectiva electoral (Delicio et al., 2001); o también como marco institucional para explicar la eficiencia técnica de universidades de gestión pública (Coria, 2008). Por esta razón, el trabajo que sigue se propone desarrollar un modelo cuantitativo de gobierno de una universidad nacional típica que explique el comportamiento del CS o los CD. Aclaremos que el objetivo del trabajo es la formalización matemática de los intereses de cada grupo de interés del gobierno universitario y del rol de un instrumento normativo en particular, el reglamento de concursos de profesor, que a nuestro juicio es fundamental para entender la gobernanza de la Universidad.

\section{La Universidad típica.}

Consideremos una universidad (podría ser también una facultad o una unidad académica menor) gobernada por un Consejo Académico de profesores, graduados y alumnos, en una relación tal que al menos la mitad de los consejeros sean profesores y la otra mitad se distribuya equitativamente entre graduados y alumnos. En este Consejo las decisiones se toman por mayoría simple, es decir, con acuerdo de la mitad más uno de los consejeros, sin que existan mecanismos especiales de desempate. 
Si bien estos mecanismos existen en todos los órganos de gobierno, como el voto del rector o decano, su uso reiterado suele acarrear "costos políticos" tan altos que en la práctica tienden a evitarse.

Supongamos, además, que los consejeros de cada claustro representan los intereses de sus pares $\mathrm{y}$, salvo por el hecho de ser consejeros, no se distinguen en nada respecto de ellos. En otras palabras, los consejeros no tienen intereses propios distintos de los de sus representados, y no hay obstáculo político alguno para que cualquiera de los representados pueda ser electo representante.

Los profesores de esta Universidad tienen a su cargo la enseñanza en todos sus aspectos (incluyendo la gestión administrativa de la propia institución) y se designan por concurso de antecedentes y oposición (Llamamos antecedentes a los méritos científicos y académicos del postulante y prueba de oposición a la clase pública orientada a demostrar su destreza didáctica). En los concursos se evalúa a los profesores tanto por su desempeño docente como por su producción científica (por producción científica nos referimos a publicaciones con referato, presentaciones en congresos, edición de libros, dictado de conferencias y seminarios, etc.). Las designaciones son por tiempo limitado, aunque el cargo puede renovarse indefinidamente, pero siempre por concurso. Los profesores tienen una relación laboral de dedicación exclusiva con la Universidad y por la especificidad de su formación sólo pueden ejercer laboralmente en el ámbito educativo, es decir, no alternan la actividad docente con el ejercicio libre de la profesión. Por su conocimiento de la gestión educativa, son los profesores (a través del rector o decano) quienes presentan la gran mayoría de las iniciativas al Consejo. El claustro de profesores puede estar integrado únicamente por profesores, $\mathrm{o}$ por profesores y docentes auxiliares, como ocurre en algunas universidades nacionales. A los fines del trabajo, la distinción entre jerarquías docentes es irrelevante ya que ambas son corresponsables de la enseñanza y se hallan sujetas a evaluaciones periódicas de mérito académico. En otras palabras, ambas jerarquías integran el mismo grupo de interés.

Los graduados son profesionales libres y no tienen otro vínculo con la Universidad más allá del título profesional otorgado por ella. Esto significa que los graduados no mantienen vínculos de estudio o trabajo con la Universidad (aunque la omisión de estudios de postgrado pueda parecer poco realista, la realidad muestra que la proporción de graduados que siguen estudios de postgrado es baja). Los graduados enfrentan, además, un mercado laboral plenamente informado del prestigio académico actual de la Universidad, de modo que sus posibilidades laborales, su nivel remunerativo e incluso su prestigio profesional dependen directamente del prestigio de la Universidad. En elámbito del Consejo, los graduados actúan en función de sus intereses de claustro e independientemente de la opinión de los profesores y los estudiantes. Este perfil de claustro es, en general, realista, incluso en aquellas universidades en que los docentes auxiliares son parte del claustro de graduados ya que su peso en el padrón del claustro suele ser reducido. No obstante, no descartamos la existencia de unidades académicas atípicas en las que nuestro perfil no sea representativo.

Los estudiantes asisten a la Universidad únicamente a tomar cursos en la temática de la carrera en que se inscribieron, es decir, excluimos otras motivaciones como las deportivas, de pertenencia a un cierto grupo social, o motivaciones políticas, por ejemplo. Los estudiantes no reciben estipendio alguno de la Universidad, ni mantienen con ella relaciones laborales de ningún tipo. La Universidad es libre de aranceles, o éstos son tan bajos que no representan un obstáculo para estudiar, y no existen otros impedimentos para el estudio (de infraestructura, disponibilidad de recursos tecnológicos, bibliográficos, etc.) que no sea la inasistencia a clase. Este supuesto podría reemplazarse por otro que asegure una amplia disponibilidad de becas de estudio. No existen tampoco limitantes debidas a la formación en etapas educativas previas debido a que operan mecanismos de selección de ingreso como el descripto por (Hernández, 2019). Por lo tanto, los estudiantes son alumnos altamente motivados y, en ausencia de otras limitantes, su desempeño académico depende directamente del esfuerzo pedagógico docente. Por "esfuerzo pedagógico docente" nos referimos a un conjunto de acciones orientadas a brindar una enseñanza de alta calidad, como redacción de material de estudio, preparación de trabajos prácticos y de clases teóricas, atención de 
consultas fuera del horario de clases, redacción y corrección de exámenes, etc. Este conjunto de acciones, que es necesariamente multidimensional, puede resumirse en una variable latente mediante una construcción similar al "modelo formativo" de calidad de servicio de docencia de (Valdivieso, 2013). Al igual que los graduados, los estudiantes actúan en el Consejo según sus propios intereses, independientemente de la opinión de los otros claustros.

Omitimos de esta caracterización la representación del personal no docente, recientemente incorporado en los consejos directivos. Omitimos caracterizarla por dos razones: la primera, que el cuerpo no docente no reviste la categoría de "claustro" de acuerdo a los estatutos universitarios $\mathrm{y}$, por lo tanto, no integra el núcleo fundacional de grupos de interés de la universidad reformista. La segunda, y quizá más importante, porque en muchos casos los representantes no docentes tienen voz, pero no voto en los consejos directivos, y aun teniendo derecho a voto prácticamente no intervienen en la discusión de cuestiones académicas como las que trataremos en el trabajo. Al igual que en el claustro de graduados, no descartamos que haya unidades académicas atípicas que desmientan esta afirmación.

En esta Universidad el único recurso relevante para el cumplimiento de sus objetivos es el recurso humano, ya que los recursos materiales no son limitantes. Aunque este supuesto parezca poco realista, en verdad, el $85 \%$ del presupuesto de la UBA, por ejemplo, corresponde a salarios y sólo el $15 \%$ a gastos de funcionamiento. La cantidad de profesores se determina exógenamente a la Universidad, por ejemplo, a través de un presupuesto aprobado en instancias superiores de gobierno como el Congreso Nacional. La cantidad de alumnos también se define exógenamente, por ejemplo, por motivaciones vocacionales o perspectivas laborales futuras y no puede ser alterada por iniciativa del Consejo Académico.

La Universidad descripta más arriba representa un esquema organizativo que en Argentina se conoce como de "universidad reformista", en alusión a la Reforma Universitaria de 1918, o "universidad tradicional" en términos de (Nosiglia \& Mulle, 2015). No discutiremos aquí hasta qué punto la Universidad actual continúa esta tradición, aunque en términos generales pareciera que este esquema ha perdurado más en Facultades de humanidades que en Facultades de ciencias naturales.

\section{La función de utilidad del docente.}

Consideremos ahora una función de utilidad individual compuesta por dos términos, uno que representa la utilidad obtenida por la producción científica, y otro que representa la utilidad obtenida por dedicación a la enseñanza. La suma ponderada de ambos términos constituye la utilidad total del docente. Esta utilidad podría interpretarse como un índice de mérito académico similar al utilizado para calificar postulantes en concursos docentes. Cada término del índice es el resultado de multiplicar el puntaje máximo previsto para cada tipo de actividad docente (enseñanza o investigación) por un factor, definido en el intervalo $[0 ; 1]$, que es función de la dedicación del docente a dicha actividad. Interpretado de este modo, el problema del docente consiste en optimizar la dedicación a cada tipo de actividad, de manera de alcanzar el máximo puntaje en el concurso. Expresado matemáticamente, la función de utilidad es:

$$
U\left(x_{1}, x_{2}\right)=\theta_{1} \frac{\ln x_{1}+1}{\ln 2}+\theta_{2} x_{2} \quad \text { s.a. } \quad x_{1}+x_{2}=1, \quad 0 \leq x_{1} \leq 1,0 \leq x_{2} \leq 1,
$$

donde $x_{1}$ es la fracción de tiempo dedicado a la investigación y $x_{2}$ es la fracción de tiempo dedicado a docencia, tanto presencial frente a alumnos como no presencial. Los ponderadores $\theta_{1}$ y $\theta_{2}$ son los puntajes máximos previstos en el reglamento de concursos para cada tipo de actividad y la constante $c t e .=\ln 2$ es simplemente una constante de escala que normaliza la función entre 0 y 1 . Nótese que bajo esta especificación la utilidad marginal de la investigación es decreciente, 
pero la utilidad marginal de la enseñanza es constante. Luego, para optimizar esta función de utilidad, planteamos el lagrangeano:

$$
L\left(x_{1}, x_{2}, \lambda\right)=\theta_{1} \frac{\ln x_{1}+1}{\ln 2}+\theta_{2} x_{2}+\lambda\left(x_{1}+x_{2}-1\right)
$$

donde $\lambda$ es un multiplicador de Lagrange. Las condiciones de primer orden son:

$$
\frac{\partial L}{\partial x_{1}^{*}}=\frac{\theta_{1}}{\ln 2} \frac{1}{x_{1}^{*}+1}-\lambda^{*}=0, \quad \frac{\partial L}{\partial x_{2}^{*}}=\theta_{2}+\lambda^{*}=0, \quad y \quad \frac{\partial L}{\partial \lambda^{*}}=x_{1}^{*}+x_{2}^{*}-1=0,
$$

y a partir de las mismas hallamos el punto crítico $\left(x_{1}^{*}, x_{2}^{*}\right)$, donde:

$$
x_{1}^{*}=\frac{1}{\ln 2}\left(\frac{\theta_{1}}{\theta_{2}}\right)-1, \quad y \quad x_{2}^{*}=2-\frac{1}{\ln 2}\left(\frac{\theta_{1}}{\theta_{2}}\right) .
$$

La utilidad en este punto es:

$$
U_{\max }=\frac{\theta_{1}}{\ln 2} \ln \left\lceil\frac{1}{\ln 2}\left(\frac{\theta_{1}}{\theta_{2}}\right)\right\rceil+\theta_{2}\left[2-\frac{1}{\ln 2}\left(\frac{\theta_{1}}{\theta_{2}}\right)\right\rceil .
$$

El punto $\left(x_{1}^{*}, x_{2}^{*}\right)$ corresponde claramente a un máximo de la función $L\left(x_{1}^{*}, x_{2}^{*}, \lambda\right)$, ya que satisface las condiciones de segundo orden para un máximo:

$$
\frac{\partial^{2} L}{\partial x_{1}^{2}}=-\frac{\theta_{1}}{\ln 2} \frac{1}{\left(\ln x_{1}+1\right)^{2}} \leq 0, \frac{\partial^{2} L}{\partial x_{2}^{2}}=0, \quad y \quad \frac{\partial^{2} L}{\partial \lambda^{2}}=0
$$

El resultado 2 sugiere, además, la siguiente cota (impuesta por las restricciones $x_{1}^{*}, x_{2}^{*} \in[0 ; 1]$ ) para el cociente de puntajes máximos:

$$
\ln 2 \leq \frac{\theta_{1}}{\theta_{2}} \leq 2 \ln 2 .
$$

En este modelo, la asignación de tiempo para cada tipo de actividad queda totalmente a cargo del docente. No obstante, la Universidad podría (de hecho, en la práctica, lo hace) establecer dedicaciones mínimas para alguna de las actividades, normalmente la enseñanza. Si se estableciera una dedicación mínima para la enseñanza, llamémosla $\tilde{x}_{2}$, la solución del problema de optimización sería una función partida, en la que las soluciones $x_{1}^{*} y x_{2}^{*}$ serían las mismas en el tramo $x_{2}^{*}>\tilde{x}_{2}$, pero $x_{2}^{*}=\tilde{x}_{2}$ y $x_{1}^{*}=1-\tilde{x}_{2}$ si $x_{2}^{*} \leq \tilde{x}_{2}$ en el tramo $x_{2}^{*} \leq \tilde{x}_{2}$. La introducción de unadedicación mínima la docencia reduce la cota superior del cociente de puntajes máximos a

$$
\ln 2 \leq \frac{\theta_{1}}{\theta_{2}} \leq\left(2-\tilde{x}_{2}\right) \ln 2 .
$$

En este punto conviene hacer una breve digresión para analizar a la luz de la función de utilidad algunos comportamientos docentes observados en la práctica:

(a) Si se relaja la restricción $x_{1}+x_{2}=1$, es decir, si la dedicación laboral del docente es completamente flexible, el docente simplemente maximiza la función de utilidad:

$$
U^{\prime}\left(x_{1}, x_{2}\right)=\theta_{1} \frac{\ln x_{1}+1}{\ln 2}+\theta_{2} x_{2}-\left(x_{1}+x_{2}\right) \quad \text { s.a. } x_{1} \geq 0, x_{2} \geq 0 .
$$

En este contexto, si bien no se obliga al docente a distribuir toda su dedicación entre docencia e investigación, la dedicación a estas actividades reduce su tiempo libre operando $x_{1}+x_{2}$ como un "costo". La función $U^{\prime}\left(x_{1}, x_{2}\right)$ será máxima en: 


$$
x_{1}^{*}=\frac{\theta_{1}}{\ln 2}-1, \quad y \quad \theta_{2}^{*}=1
$$

Es decir, la dedicación a la docencia $x_{2}$ es independiente del puntaje máximo asignado a este concepto y a la investigación, aunque $\theta_{2}$ debe ser igual a la unidad para que exista una situación de optimalidad. Notemos, sin embargo, que la utilidad máxima en este caso es:

$$
U_{\text {max }}^{\prime}=\frac{\theta_{1}}{\ln 2} \ln \left(\frac{\theta_{1}}{\ln 2}\right)+\theta_{2} x_{2}-\left(\frac{\theta_{1}}{\ln 2}-1+x_{2}\right) .
$$

o bien

$$
U_{\text {max }}^{\prime}=U_{\max }+\frac{\theta_{1} \ln \theta_{2}}{\ln 2}+\left(\theta_{2}-1\right) x_{2}-2 \theta_{2}+1
$$

Esta última expresión pone de manifiesto la ambigüedad del relajamiento de la restricción $x_{1}+x_{2}=1$ sobre la utilidad máxima, ya que $U_{\max }^{\prime}$ será mayor o menor que $U_{\max }$ dependiendo de los valores que tomen $\theta_{1}, \theta_{2}$ y $x_{2}$.

(b) Si la Universidad establece una dedicación mínima a la enseñanza $\left(\tilde{x}_{2}\right)$ aunque el docente puede distribuir libremente su tiempo entre enseñanza e investigación una vez cumplida la dedicación mínima a la enseñanza, la solución para $x_{1}$ y $x_{2}$ es una función partida que vale:

$$
x_{2}^{*}=\tilde{x}_{2}, x_{1}^{*}=1-\tilde{x}_{2} \text { si } \ln 2 \leq \frac{\theta_{1}}{\theta_{2}} \leq\left(2-\tilde{x}_{2}\right) \ln 2
$$

o bien, la solución (2) si:

$$
\left(2-\tilde{x}_{2}\right) \ln 2 \leq \frac{\theta_{1}}{\theta_{2}} \leq 2 \ln 2 .
$$

(c) La relación de puntajes máximos cambia a lo largo de la carrera docente, por ejemplo, si los puntajes máximos en concursos de renovación fueran distintos a los de concursos para nuevos cargos. Para analizar este caso supongamos por simplicidad que el docente prevé concursar para acceder a un nuevo cargo y renovarlo una sola vez antes de jubilarse. En este caso, la función de utilidad actual es igual al valor presente de las utilidades futuras, es decir,

$$
U\left(x_{1}, x_{2}\right)=U^{I}\left(x_{1}, x_{2}\right)+\delta U^{I I}\left(x_{1}, x_{2}\right)
$$

donde $\delta$ es un factor de descuento en el intervalo $(0 ; 1]$ indicativo del peso que el docente le asigna lasutilidades futuras, el supraíndice Iserefiere al primerperíodoy el supraíndice II al segundo período. Procediendo como en los puntos anteriores, las condiciones de primer orden son:

$$
\frac{\partial U\left(x_{1}, x_{2}\right)}{\partial x_{j}}=\frac{\partial U^{I}\left(x_{1}, x_{2}\right)}{\partial x_{j}}+\delta \frac{\partial U^{I I}\left(x_{1}, x_{2}\right)}{\partial x_{j}}=0, \text { para todo } j=\{1,2\}
$$

y la solución:

$$
x_{1}^{*}=\frac{1}{\ln 2}\left(\frac{\theta_{1}{ }^{I}+\delta \theta_{1}{ }^{I I}}{\theta_{2}{ }^{I}+\delta \theta_{2}{ }^{I I}}\right)-1, \quad y x_{2}^{*}=2-\frac{1}{\ln 2}\left(\frac{\theta_{1}{ }^{I}+\delta \theta_{1}{ }^{I I}}{{\theta_{2}}^{I}+\delta \theta_{2}{ }^{I I}}\right), \quad 0<\delta \leq 1,
$$

donde vemos que la solución óptima depende no sólo de la relación de puntajes futura, sino también del factor de descuento $\delta$. Lógicamente, este factorserá menorcuanto mayor sea el 
plazo entre concursos. Si la relación de puntajes se mantuviera constante, las dedicaciones óptimas no cambiarían con el tiempo, independientemente de cuál sea el plazo entre llamados a concurso.

La solución intertemporal sugiere también que si un profesor tiene expectativa (o, mejor aún, una oferta concreta) de continuar enseñando después del retiro, reducirá gradualmente la dedicación a la investigación e incrementará la dedicación a la enseñanza. Formalmente, la dedicación óptima a lo largo del tiempo será:

$$
x_{1}^{*}(t)=\frac{1}{\ln 2}\left(\frac{1+\sum_{i=1}^{t-1} \delta^{i}}{1+\sum_{i=1}^{t+r} \delta^{i}}\right)\left(\frac{\theta_{1}}{\theta_{2}}\right)-1, \quad y \quad x_{2}^{*}(t)=2-\frac{1}{\ln 2}\left(\frac{1+\sum_{i=1}^{t-1} \delta^{i}}{1+\sum_{i=1}^{t+r} \delta^{i}}\right)\left(\frac{\theta_{1}}{\theta_{2}}\right) .
$$

donde $t$ es la cantidad de períodos hasta la jubilación y $r$ es la cantidad de períodos de ejercicio docente posteriores a la jubilación. Aclaremos que $t$ representa, en rigor, la cantidad de períodos entre concursos, por lo cual cuanto menor sea el período de designación, más suave serála curva de $x_{2}^{*}(t)$ en función de $t$. Por el contrario, cuánto mayor sea el período entre concursos, más escalonada será la curva de $x_{2}^{*}(t)$. De todos modos, el comportamiento de $x_{2}^{*}(t)$ dependerá fuertemente de las condiciones de contratación del profesory delatradición institucional respecto a la permanencia de los profesores pasada la edad de retiro. En situaciones normales, la oferta de permanencia se revela al final de la carrera docente, de modo que bien cabría esperar que durante la mayor parte de su vida laboral, el profesor fuera indiferente ante la posibilidad permanecer (asumiera que $r \approx 0$ ) y que recién al final de su carrera aumentara la dedicación a la enseñanza.

\section{La función de costo político del Consejo Académico.}

La composición del Consejo Académico requiere que toda resolución cuente con el apoyo de al menos un consejero graduado o estudiantil, o de al menos un profesor si hubiera acuerdo entre estudiantes y graduados. Es decir, que la gobernabilidad, y en última instancia la gobernanza, de la institución dependen completamente de un mínimo acuerdo entre claustros. En consecuencia, los consejeros profesores tienen la necesidad de conciliar los intereses de graduados y alumnos para gobernar la Universidad. Matemáticamente esto equivale a minimizar una función de distancia $\Delta$ entre la demanda de docencia de los estudiantes y la demanda de producción científica de los graduados. Esta función, a la que llamaremos $g(\Delta)$ o función de costo político es alguna función $g: \mathbb{R} \rightarrow \mathbb{R}^{+}$de la diferencia entre la dedicación total a la enseñanza y la dedicación total a la investigación sujeta a la condición de optimalidad de la utilidad docente, deducida más arriba. Justificaremos estas funciones más adelante. Formalmente, si no se imponen dedicaciones mínimas a la enseñanza, una forma sencilla para $g(\Delta)$ es:

$$
g(\Delta)=\left[\sum_{i=1}^{n} x_{2}^{*}-\sum_{i=1}^{n} x_{1}^{*}\right]^{2}=n^{2}\left\{\left[2-\frac{1}{\ln 2}\left(\frac{\theta_{1}}{\theta_{2}}\right)\right]-\left[\frac{1}{\ln 2}\left(\frac{\theta_{1}}{\theta_{2}}\right)-1\right]\right\}^{2}
$$

según el criterio de mínimos cuadrados. En este caso, el problema de optimización es:

$$
\min _{\theta_{1} / \theta_{2}}\left\{n^{2}\left[3-\frac{2}{\ln 2}\left(\frac{\theta_{1}}{\theta_{2}}\right)\right]^{2}\right\}
$$


donde la relación entre puntajes máximos es la variable respecto de la que se optimiza la función de costo político ya que, como dijimos más arriba, consideramos esta relación contenida en el reglamento de provisión de cargos de profesor, el principal instrumento normativo del Consejo. Siguiendo el procedimiento usual de optimización, hallamos que $g(\Delta)$ es mínima en el punto:

$$
\frac{\theta_{1}^{*}}{\theta_{2}{ }^{*}}=\frac{3}{2} \ln 2 \approx 1,04
$$

La verificación de la condición de segundo orden revela que en este punto la función es, efectivamente, mínima ya que:

$$
\frac{\partial^{2} \Delta^{2}}{\partial\left(\theta_{1} / \theta_{2}\right)^{2}}=\frac{4 n^{2}}{(\ln 2)^{2}}>0
$$

La figura 1 muestra tres funciones de utilidad $U\left(x_{1}, x_{2} \mid \theta_{2}=1\right)$ para $\theta_{1}=\{0,85 ; 1,04 ; 1,15\} \mathrm{y}$ superpuestas con éstas la función de utilidades máximas $U_{\max }=f\left(x_{1}^{*} x_{2}^{*}\right)$ y la función de costo político, $C=g\left(x_{1}^{*} x_{2}^{*}\right)$, en sus correspondientes escalas. Tanto $U_{\max }$ como $C$ son funciones de $x_{1}^{*} \mathrm{y}$ $x_{2}^{*}$ que a su vez son función de la relación de puntajes $\theta_{1} / \theta_{2}$ ). Esta relación se halla implícita en el gráfico porque omitimos agregar una escala adicional para facilitar la lectura. Se puede observar que en el punto $x_{1}=x_{2}=0,5$ y $\theta_{1} / \theta_{2}=1,04$, la utilidad docente es máxima y el costo político del Consejo es mínimo, siendo éste el punto de máxima gobernabilidad institucional. En términos propios del Consejo, éste es el punto en el que los tres claustros alcanzan consenso. El resultado (5) tiene algunas implicancias que conviene destacar:

a. La relación óptima de puntajes es independiente de la cantidad de docentes $n$ de la Universidad y, por transitividad, de las decisiones presupuestarias que se tomen en niveles superiores de gobierno. Incluso, si extendiéramos el modelo a dos períodos, en el que la cantidad de docentes del segundo período fuera distinta a la del primer período, la conclusión sería la misma. Formalmente, planteando:

$$
\min _{\theta_{1} / \theta_{2}}\left\{n_{1}^{2}\left[3-\frac{2}{\ln 2}\left(\frac{\theta_{1}}{\theta_{2}}\right)\right]^{2}+n_{2}^{2}\left[3-\frac{2}{\ln 2}\left(\frac{\theta_{1}}{\theta_{2}}\right)\right]^{2}\right\}
$$

se aprecia fácilmente que $n_{1}$ y $n_{2}$ operan como constantes de proporcionalidad que se cancelan al plantear las condiciones de primer orden:

$$
\frac{\partial}{\partial\left(\theta_{1}{ }^{*} / \theta_{2}{ }^{*}\right)}\left[g\left(\Delta_{1}\right)+g\left(\Delta_{2}\right)\right]=-2\left(n_{1}^{2}+n_{2}^{2}\right)\left[3-\frac{2}{\ln 2}\left(\frac{\theta^{*}}{\theta_{2}{ }^{*}}\right)\right]\left(\frac{2}{\ln 2}\right)=0
$$

b. La relación óptima de puntajes que surge del acuerdo entre claustros conduce a una distribución equitativa de la dedicación docente a la enseñanza y a la investigación, ya que reemplazando la relaciónóptima $\theta_{1}{ }^{*} / \theta_{2}{ }^{*}$ en las soluciones individuales $x_{1}^{*}$ y $x_{2}^{*}$ resulta:

$$
x_{1}^{*}=x_{2}^{*}=\frac{1}{2}
$$

aun cuando la relación $\theta_{1} / \theta_{2}$ sea levemente favorable a la producción científica. Esta distribución de dedicaciones cambia si se ponderara cada tipo de actividad en la función de costo político con algún criterio arbitrario. En ese caso,

$$
g(\Delta)=n^{2}\left\{w\left[2-\frac{1}{\ln 2}\left(\frac{\theta_{1}}{\theta_{2}}\right)\right]-(1-w)\left[\frac{1}{\ln 2}\left(\frac{\theta_{1}}{\theta_{2}}\right)-1\right]\right\}^{2},
$$


y la solución para el cociente de puntajes resulta:

$$
\frac{\theta_{1}^{*}}{\theta_{2}{ }^{*}}=(1+w) \ln 2
$$

Esta expresión es claramente una extensión de la solución (5) y podría ser de utilidad para representar el caso de un Consejo en el que los graduados (o los estudiantes) tuvieran mayor representación que los estudiantes (o graduados), pero manteniendo en $50 \%$ la representación de profesores. Nótese que a medida que el factor de ponderación $w$ aumenta, también debe aumentar la relación $\theta_{1}{ }^{*} / \theta_{2}{ }^{*}$ para garantizar la gobernabilidad de la institución.

c. Las dedicaciones óptimas a la investigación y la docencia $x_{1}^{*}$ y $x_{2}^{*}$ respectivamente, son proporcionales a la constante de normalización cte. $=\ln 2$. Si se produjera un shock en la productividad científica, sin que el Consejo actualizara el reglamento de concursos, es decir, manteniendo constante la relación $\theta_{1}{ }^{*} / \theta_{2}{ }^{*}$, cabría esperar un shock en el mismo sentido en $x_{1}^{*} \mathrm{y}$ en sentido opuesto en $x_{2}^{*}$. Si, por el contrario, el consejo monitoreara permanentemente la productividad científica y ajustara la relación $\theta_{1}{ }^{*} / \theta_{2}{ }^{*}$ proporcionalmente a la misma, las dedicaciones $x_{1}^{*}$ y $x_{2}^{*}$ se mantendrían constantes a lo largo del tiempo.

Figura 1. Dedicación a la enseñanza $x_{2}$ que garantiza el máximo mérito académico docente y el mínimo costo político en el Consejo.

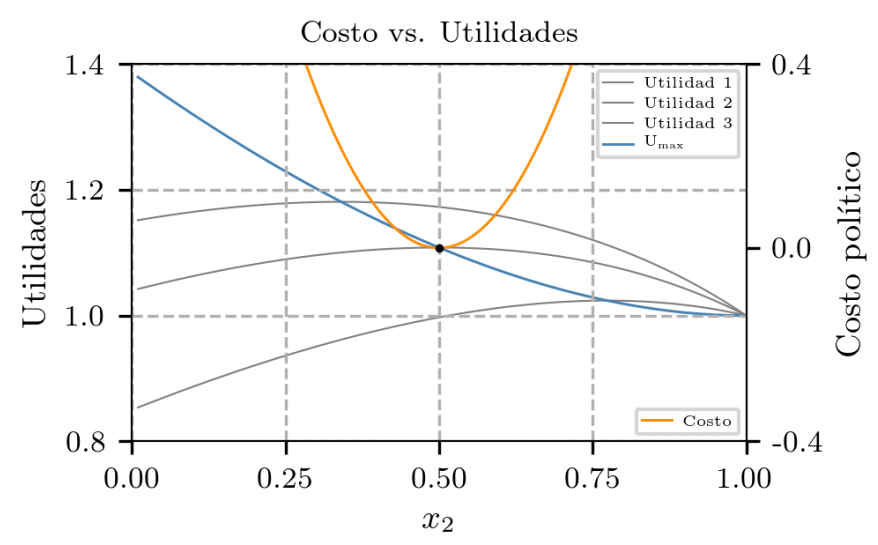

Fuente: Elaboración propia.

d. Siseestablece una dedicación mínimaalaenseñanza, larelación depuntajesóptimasería función de ésta. En este caso la función a minimizar sería la función partida:

$$
\min _{\theta_{1} / \theta_{2}}\left\{n^{2}\left[3-\frac{2}{\ln 2}\left(\frac{\theta_{1}}{\theta_{2}}\right)\right]^{2} ; n_{2}^{2}\left[\tilde{x}_{2}-\frac{\theta_{1}}{\ln 2}+1\right]^{2}\right\}
$$

Recordemos que al imponer $x_{2}=\tilde{x}_{2}$, el puntaje para enseñanza resultaba $\theta_{2}=1$. Luego, si $x_{2}=\tilde{x}_{2}$, la solución es:

$$
\frac{\theta_{1}{ }^{*}}{\theta_{2}{ }^{*}}=\left(1+\tilde{x}_{2}\right) \ln 2 .
$$




\section{Conclusión.}

El gobierno de las universidades nacionales puede representarse razonablemente a través de un modelo de stackeholders o grupos de interés llamados “claustros". El claustro de profesores estáconstituido por la planta docente de la Universidad, cuya función es, no sólo impartir enseñanza, sino administrar la propia Universidad. En términos corporativos, los profesores constituyen tanto el staff, como la gerencia de una organización. Aclaremos que si bien recurrimos con frecuencia a términos propios de la bibliografía sobre gobierno de las organizaciones, ello no implica en modo alguno que equiparemos universidad pública con empresa como organizaciones equivalentes.

Los profesores enfrentan un mercado laboral reducidoy altamente especializado, por lo que su principal interés es garantizar su continuidad laboral perpetuando la Universidad. El claustro de graduados reúne al conjunto de egresados de la Universidad, quienes pueden ser interpretados como tenedores de un título o bono que les proporciona una renta proporcional al prestigio actual de la Universidad y a los alcances profesionales del propio título. Para revalorizar permanentemente el título profesional, la Universidad debe generar nuevos conocimientos que la posicionen en los puestos más elevados de los rankings académicos y también que expandan el campo laboral de sus egresados. Los estudiantes (el tercer claustro) son los usuarios de la Universidad, interpretada como institución prestadora de un servicio educativo, y esperan recibir de ella una educación de buena calidad. Satisfaciendo las demandas de estudiantes y graduados, los profesores garantizan la gobernabilidad de la Universidad.

La Universidad de nuestro modelo está gobernada por un Consejo Académico que satisface las demandas de estudiantes y graduados mediante incentivos adecuados establecidos en el reglamento de provisión de cargos de profesor o mediante resoluciones explícitas sobre dedicaciones mínimas a la enseñanza. El reglamento de provisión de cargos es un instrumento complejo que opera sobre la dedicación a la enseñanza y la investigación principalmente a través de un esquema de puntajes máximos para cada una de estas actividades, y ciertas condiciones de renovación periódica de cátedras y plazos de designación. Nuestro estudio se focaliza en la relación de puntajes máximos, y concluye que existe una relación óptima de puntajes levemente superior a 1 (para la función de mérito académico utilizada) que garantiza el cumplimiento de los objetivos institucionales antes mencionados y por ende la gobernabilidad de la organización. Esta relación depende de la relación de representación de cada claustro en el Consejo, de la productividad científica, y de la existencia de dedicaciones mínimas a la enseñanza. Respecto a este último punto, nuestro modelo desestima la eficacia de las dedicaciones mínimas a la enseñanza como instrumento de promoción de la enseñanza, en primer lugar, porque es posible alcanzar la misma dedicación a la enseñanza simplemente ajustando de la relación de puntajes; en segundo lugar, porque las dedicaciones mínimas observadas en la práctica se hallan muy por debajo de la dedicación óptima $x_{2}^{*} \mathrm{y}$, en tercer lugar, porque las dedicaciones mínimas por encima de $x_{2}^{*}$ serían políticamente costosas en el ámbito del Consejo. Nótese, por ejemplo, que el costo político (fig.1) de aprobar una dedicación mínima de $\tilde{x}_{2}=0,75$ sería altísimo. El modelo muestra, además, que los shocks presupuestarios no afectan mayormente la gobernanza de la Universidad, ni las actividades científicas y docentes, sino simplemente el tamaño de la plantilla docente. La cantidad de alumnos tampoco afecta la gobernanza en este sencillo modelo.

La simplicidad del modelo no debería dejar la falsa impresión de que la única función del Consejo Académico deunauniversidad es ajustarperiódicamente larelación depuntajes máximos en el reglamento de concursos docentes. Por el contrario, el Consejo tiene también la misión de garantizar las condiciones de gobernabilidad implícitas en el modelo, es decir, los llamados "supuestos" del modelo. Entre ellos, es crucial que el Consejo (a) procure que los intereses de profesores, graduados y estudiantes no se superpongan, por ejemplo mediante la aprobación de normas de incompatibilidad laboral, como la prevista en el art. 53 inc. d de la ley 24.521 de Educación Superior; (b) monitoree permanentemente tasa media de publicación docente, $u$ otra variable proxy de la productividad científica, a fin de captar cambios en la función de producción, que como sabemos es inobservable; y (c) promueva evaluaciones externas de calidad institucional 
para contar con medidas objetivas de calidad educativa y excelencia académica demandas por estudiantes y graduados, respectivamente.

Conviene advertir al lector sobre los peligros de interpretar al modelo como un modelo integral de gobierno universitario. El modelo no prevé, por ejemplo, cómo la gestión de recursos materiales, o la competencia con otras casas de estudio por la captación de alumnos, o por prestigio académico, influyen en el gobierno universitario. Esta omisión no necesariamente convierte al modelo en uno falso o defectuoso, sino que lo restringe al campo explicativo de aquellos aspectos del gobierno universitario que nos interesaron estudiar, básicamente el rol del reglamento de provisión de cargos de profesor en la gobernanza institucional.

\section{Referencias}

Buchbinder, P. (2010). Historia de las Universidades Argentinas. Buenos Aires, Argentina: Editorial Sudamericana.

CONEAU (2007). Guía de autoevaluación. Carreras de primera fase. Resolución 1232/01. acreditación de carreras de grado de ingeniería. Comisión Nacional de Evaluación y Acreditación Universitaria. Recuperado de http://www.coneau.gob.ar.

Consejo de Universidades (2015). Régimen de Organización de Carreras, Otorgamiento de titulos $y$ Expedición de Diplomas. Recuperado de http://portales.educacion.gov.ar/spu/normativa-emanada- por-el-cuerpo/.

Convención Constituyente (1994). Constitución Nacional. Sancionada en 1853 con las reformas de los años 1860, 1866, 1898, 1957 y 1994. Recuperado de http://servicios.infoleg.gob.ar.

Coria, M. (2008). Eficiencia técnica de las universidades de gestión estatal en Argentina. Documento de Trabajo, 27. Pontificia Universidad Católica Argentina "Santa María de los Buenos Aires". Facultad de Ciencias Sociales y Económicas, Departamento de Economía.

CSJN (1996). Monges, Analía M. c/ Universidad de Buenos Aires. Fallo del 26 de diciembre de 1996. Corte Suprema de Justicia de la Nación.

Delicio, F., Carboni, S., Maestromey, M., \& Magnoler, G. (2001). El problema del gobierno universitario y la elección directa de autoridades. Universidad Nacional de Mar del Plata, Argentina. II Coloquio Internacional de Gestión Universitaria en América del Sur.

Fernández, N. (2002). La Educación Superior en la Argentina. Instituto Internacional para la Educación Superior en América Latina y el Caribe (IESALC/UNESCO) y Ministerio de Educación, Ciencia y Tecnología de la República Argentina. Recuperado de http://unesdoc.unesco.org/images/0014/001494/149464so.pdf.

García, H. (1995). La autonomía de los municipios provinciales en la reforma constitucional de 1994. Academia Nacional de Ciencias Morales y Políticas. Recuperado de https://www.ancmyp.org.ar.

Halperin, T. (2002). Historia de la Universidad de Buenos Aires. Buenos Aires, Argentina: Eudeba. 
Hernández, P. (2019). Desempeño Académico y Curso de Iniciación Universitaria: Un Análisis de Regresiones Discontinuas. Revista de Métodos Cuantitativos para la Economía y la Empresa, 27, 207-234.

Marquís, C. (2010). Sobre el Gobierno Universitario. Gestión Universitaria, 2(3). Recuperado de http://www.gestuniv.com.ar/gu_06/v2n3a2.htm

Molina, M.S. (2014). La autonomía institucional y académica de las universidades nacionales. evolución conceptual en la legislación y jurisprudencia argentina. Revista Iberoamericana de Educación Superior, 5(13), 66-89.

Nosiglia, M.C., \& Mulle, V. (2009). Las Transformaciones en el Gobierno de las Universidades Argentinas: Análisis de casos. Revista Argentina de Educación Superior, 1(1),173-200.

Nosiglia, M.C., \& Mulle, V. (2015). El gobierno de las instituciones universitarias a partir de la Ley de Educación Superior 24.521: un análisis de los Estatutos Universitarios. Revista Iberoamericana de Educación Superior, 15(6), 72-89.

Novelli, C. (2011). Reseña bibliográfica de: La autonomía universitaria: definiciones normativas y jurisprudenciales en clave histórica y actual. Academia. Revista sobre enseñanza del Derecho, 9(17), 261-274.

Romera, M., \& Puricelli, G. (1994). Recuperando la voz. El movimiento estudiantil en los primeros años de la transición democrática. Buenos Aires: Federación Universitaria de Buenos Aires.

Unzué, M. (2012). Historia del origen de la Universidad de Buenos Aires (A propósito de su 190 aniversario). Revista Iberoamericana de Educación Superior, 8(3), 72-88.

Valdivieso, C. (2013). Comparación de los modelos formativo, reflexivo y de antecedentes de evaluación estudiantil del servicio de docencia. Revista de Métodos Cuantitativos para la Economía y la Empresa, 16, 95-120. 